\title{
Type II Bismuth-Corlette Hilar Cholangiocarcinoma
}

National Cancer Institute

\section{Source}

National Cancer Institute. Iype II Bismuth-Corlette Hilar Cholangiocarcinoma. NCI

Thesaurus. Code C134738.

Tumor involves the confluence of the right and left hepatic ducts. (AJCC 8th ed.) 